\title{
Trade-off Evaluation for Process Configuration Scheme
}

\author{
Lin Wang ${ }^{1}$, Yongjian Zhang $^{1}$, Xuyun $\mathrm{Fu}^{1}$ and Shisheng Zhong ${ }^{2}$ \\ ${ }^{1}$ School of Naval Architecture and Ocean Engineering, Harbin Institute of Technology at Weihai, No.2 \\ Wenhuaxi Road, Weihai, China. \\ ${ }^{2}$ School of Mechatronics Engineering, Harbin Institute of Technology, No.92 Xidazhi Street, Harbin, \\ China.
}

KEY WORDS: process configuration scheme; reusability; validity; trade-off evaluation

\begin{abstract}
In order to optimize the process configuration design scheme, the trade-off evaluation for process configuration scheme based on reusability and validity was proposed. By comparative analysis between the configuration process technology and traditional process and referring to the method of product configuration plan, the reusability index based on process route similarity and effectiveness index based on product characteristics and process feature matching were given out. In this way, no extra weights would be in need when carrying though visualized evaluation and analysis of multiple process configuration plans using the reusability index and the effectiveness index as substitute. This trade-off evaluation method was validated in the case of process configuration about satellite plate machining process.
\end{abstract}

\section{INTRODCUTION}

In order to fighting in a world market, product family and mass customization have been adopted by companies. Product configuration plays a key role in both product family and mass customization $[1,2]$. Schierholt proposed the process configuration concept firstly, process configuration is the task of selecting the manufacturing processes needed to manufacture a product variant according to customer specifications from a fixed, predefined set of processes, putting these manufacturing processes into sequence, and generating production data for each process while respecting all compatibility constraints on how process may be combined and ordered [3]. Although it has been more than a decade since the process configuration concept was proposed, the studies about process configuration is very limited. Some researchers focused on production configuration of product families $[4,5]$, and the others incorporated product configuration with process planning to solve the problem $[6,7,8]$.Generally, the configuration technique includes following content: configuration modelling, representation of the configuration knowledge, solving of the configuration, evaluation and optimization for configured schemes. For the configuration technique has the property of multiple constraints and solutions, it's necessary to evaluate the configured schemes. With the application of configuration in process planning, the same problem appears, therefore, the evaluation method for process configuration scheme was studied in this article.

\section{DESCRIPTION FOR EVALUATION OF PROCESS CONFIGURATION SHCEME}

\section{Reasons for Multiple Process Configuration Schemes}

The product manufacturing features and technical requirements are firstly extracted as conditions in process configuration, and then the process rotes which match the conditions are searched for the process database, finally the process parameters in process rotes are modified according to the detail of product manufacturing features and technical requirements. If there are several process schemes for one product, 
the process schemes should be evaluated. From the above description of process configuration, several processing methods and different equipment may match one manufacturing feature. In addition, the manufacturability of the product, production capacity of the equipment, the experience of engineer, and so on are the reasons for multiple process schemes. In short, the main reasons for multiple process configuration schemes are (1) variety of the process cell for one manufacturing feature, (2) variety of the combination of different process cells, (3) multiple solutions for process configuration solving methods.

\section{Evaluation Index for Process Configuration Schemes}

Process configuration is different from traditional process planning, process configuration take full advantage of useful processes which have been used for many times in workshop. Because the process configuration depends on product history process data, and the results of the configuration must be more match the machining technical requirements of the product. Therefore, according to the evaluation of the process configuration schemes, the reusability index and validity index were proposed.

Definition 1 The reusability index for evaluation of process configuration scheme could be marked as $R$, which is used to measure the reuse level of former process in process configuration scheme. The higher reuse level, $R$ tends to 1 , the lower reuse level, $R$ tends to 0 .

Definition 2 The validity index for evaluation of process configuration scheme could be marked as $V$, which is used to measure the match level of process configuration to the target process scheme. The higher match level, $V$ tends to 1 , the lower match level, $V$ tends to 0 .

\section{EVALUATION FOR PROCESS CONFIGURATION SCHEMES}

\section{Calculation for Reusability Index}

On one hand the reusability index of process configuration scheme could be calculated by the similarity between matching process and configured process, on the other hand the reusability index could be calculated by the similarity between the target product and former product. Set the similarity between matching process and configured process as $S_{p c}$, set the similarity between target product and former product as $S_{p d}$, define the reusability of process configuration scheme as $S, R=S$, then

$\omega$ is the weighting coefficient, $0 \leq \omega \leq 1$.

$$
S=\omega \cdot S_{p c}+(1-\omega) \cdot S_{p d}
$$

The calculation of $S_{p c}$ could be refer to the method in reference [9]. For the process planning are related to the product material, shape and precision. Set the product material similarity, shape similarity and precision similarity as $S_{p d \_m}, S_{p d_{-} s}$ and $S_{p d \_p}$ separately, then

$$
S_{p d}=K_{p d \_m} \cdot S_{p d \_m}+K_{p d \_s} \cdot S_{p d \_s}+K_{p d \_p} \cdot S_{p d \_p}
$$

$K_{p d \_m}$ is the weighting coefficient of property similarity, $K_{p d_{-} s}$ is the weighting coefficient of shape similarity and $K_{p d_{-} p}$ is the weighting coefficient of precision similarity, $K_{p d_{-} m}+K_{p d_{-} s}+K_{p d_{\_} p}=1$. In the equation (2), if the material of target product is the same as the former product $S_{p d \_m}=1$, else $S_{p d \_m}=0$. The calculation of $S_{p d_{-} s}$ and $S_{p d_{-} p}$ is as follows

$$
S_{p d_{-} s}=S_{p d_{-} p}=\frac{2 \cdot N_{t p_{-} f p}}{N_{t p}+N_{f p}}
$$

$N_{t p}$ is the shape number or precision number of target product, $N_{f p}$ is the shape number or precision number former product, $N_{t p \_f p}$ is the similar shape number or precision number between target product and former product. 


\section{Calculation for Validity Index}

The validity index of process configuration scheme mainly reflect in whether the key manufacturing features of the product and the technical requirements could be mapped into the process feature in the configured scheme. Therefore, the validity index could be calculated by calculating the ratio of valid process feature in the matching process scheme. Define the whole matching rate between product feature and process feature as $\lambda, V=\lambda$, then

$$
\lambda=\frac{\sum_{i=1}^{s} \lambda_{S i}+\sum_{j=1}^{m} \lambda_{M i}+\sum_{k=1}^{t} \lambda_{T i}+\sum_{l=1}^{e} \lambda_{E l}}{s+m+t+e}
$$

$\lambda_{S}$ is the shape feature matching rate, $s$ is the number of shape feature, $\lambda_{M}$ is the material feature matching rate, $m$ is the number of material feature, $\lambda_{T}$ is the technical feature matching rate, $t$ is the number of technical feature, $\lambda_{E}$ is the precision feature matching rate, $e$ is the number of precision feature. In the configured scheme, if there exist the process feature which match the product feature, the value of $\lambda_{S}, \lambda_{M}$, $\lambda_{T}$ and $\lambda_{E}$ is 1 , otherwise the value is 0 .

\section{Trade-off Evaluation for Process Configuration Schemes}

The reusability index of process configuration scheme could be expressed as horizontal ordinate, the validity index of process configuration scheme could be expressed as vertical coordinate, the relationship between reusability index and validity index could be established as Figure 1. The perfect configured scheme should have higher reusability index and validity index.

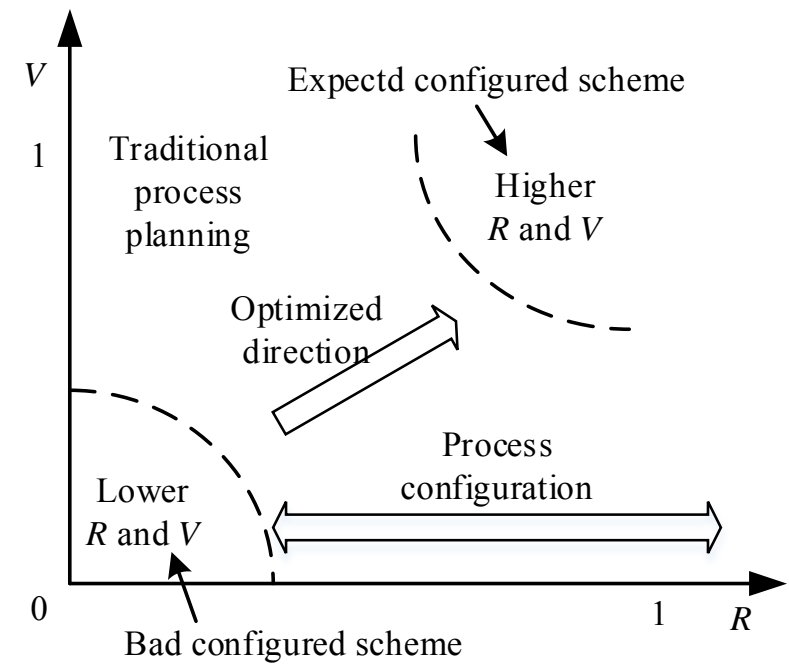

Figure 1. Trade-off evaluation graph of process configuration scheme

\section{CASE STUDY}

Satellite plate is a kind of usual component of a satellite, and honeycomb sandwich plate is one type of satellite plate which is used as force bearing structure, solar panel and outside wall of a satellite. The honeycomb sandwich plate comprises three items such as figure 2:1) two panels with high strength and thin thickness, 2) honeycomb core with light weight, and 3) adhesive which bonds the two panels and honeycomb core. 


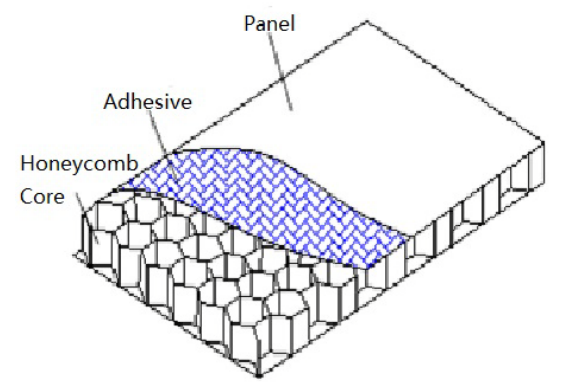

Figure 2. Basic structure of honeycomb sandwich plate

For the similar structures and functions, the satellite plates have similar production process, including panel machining process, honeycomb core machining process, embedded part machining process, embedded part assembly process, adhesive process and et al. Taking panel machining process for examples. The case data from reference [10] are used in this article. The features information of target plate and former plate, and the reusability index and validity index are as in table I. From figure 3, scheme II is the best one.

TABLE I. FEATURE INFORMATION OF TARGET PLATE AND FORMER PLATE

\begin{tabular}{|c|c|c|c|c|}
\hline features & target plate & scheme I & scheme II & scheme III \\
\hline \multirow{4}{*}{ Hole (mm) } & $4-\Phi 4.5$ & & & $8-\Phi 4.5$ \\
\hline & $9-\Phi 11.5$ & $11-\Phi 4.5$ & 11-Ф4.5 & $40-\Phi 5.5$ \\
\hline & $16-\Phi 12.2$ & $22-\Phi 5.5$ & $26-\Phi 6.8$ & $23-\Phi 12.2$ \\
\hline & $11-\Phi 27.2$ & $23-\Phi 12.5$ & $23-\Phi 10.5$ & $5-\Phi 18.5$ \\
\hline Shape $(\mathrm{mm})$ & $1200 \times 560 \times 0.3$ & $1000 \times 500 \times 0.3$ & $1000 \times 500 \times 0.3$ & $1200 \times 400 \times 0.3$ \\
\hline Material & LY12CZ & LY12CZ & LY12CZ & LY12CZ \\
\hline Tolerance & $\begin{array}{l}+0.06 \\
0\end{array}$ & $\begin{array}{l}+0.06 \\
0\end{array}$ & $\begin{array}{l}+0.06 \\
0\end{array}$ & $\begin{array}{l}+0.06 \\
0\end{array}$ \\
\hline Location & $\Phi 0.3 \mathrm{~mm}$ & $\Phi 0.3 \mathrm{~mm}$ & $\Phi 0.3 \mathrm{~mm}$ & $\Phi 0.3 \mathrm{~mm}$ \\
\hline Roughness & $R a=3.2$ & $R a=3.2$ & $R a=3.2$ & $R a=3.2$ \\
\hline $\begin{array}{c}\text { Allowance } \\
\text { (Length, Width) }\end{array}$ & $(\mathrm{L}, \mathrm{W}) \pm 0.5$ & $(\mathrm{~L}, \mathrm{~W}) \pm 0.5$ & $(\mathrm{~L}, \mathrm{~W}) \pm 0.5$ & $(\mathrm{~L}, \mathrm{~W}) \pm 0.5$ \\
\hline Requirement & \multicolumn{4}{|c|}{ no rag, no oil, no scratch } \\
\hline Reusability & & 0.921 & 0.893 & 0.887 \\
\hline Validity & & 0.685 & 0.837 & 0.809 \\
\hline
\end{tabular}

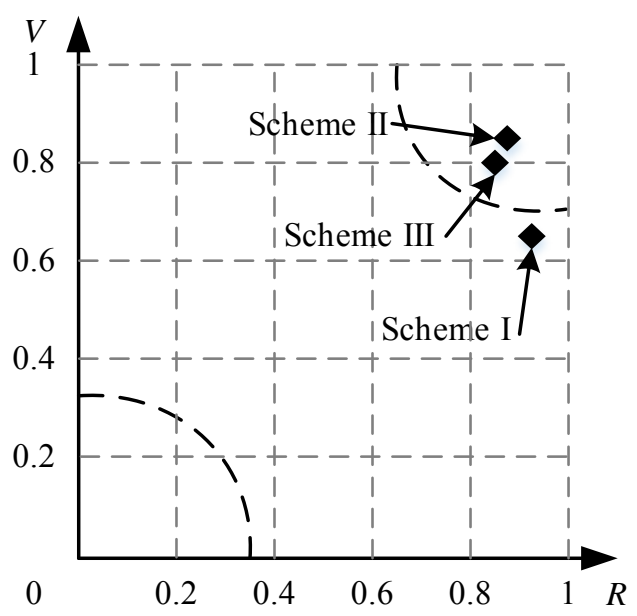

Figure 3. Trade-off evaluation graph for satellite plate machining process configuration scheme 


\section{CONCLUSION}

According to the principle of configuration technique, process configuration is the procedure to reuse and modify the history process data. With the problem of evaluation and optimization for process scheme, reusability index and validity index were proposed. The configured scheme with the higher reusability index and validity index is the optimized one in the multiple configured schemes.

\section{ACKNOWLEDGEMENTS}

This work was supported by the National High-tech Research and Development Program (863 Program) of China (NO. 2014AA041501) and the National Natural Science Foundation of China (NO.51305096).

\section{REFERENCES}

1. Jiao, J., \& Tseng, M. M. 1999. A methodology of developing product family architecture for mass customization. Journal of Intelligent Manufacturing, 10(1), 3-20.

2. Jiao, J. R., Simpson, T. W., \& Siddique, Z. 2007. Product family design and platform-based product development: a state-of-the-art review. Journal of Intelligent Manufacturing, 18(1), 5-29.

3. Schierholt, K. 2001. Process configuration: Combining the principles of product configuration and process planning. AI EDAM, 15(05), 411-424.

4. Zhang, L. L., Xu, Q., Yu, Y., \& Jiao, R. J. 2012. Domain-based production configuration with constraint satisfaction. International Journal of Production Research, 50(24), 7149-7166.

5. Zhang, L., \& Rodrigues, B. 2010. Nested coloured timed Petri nets for production configuration of product families. International Journal of Production Research, 48(6), 1805-1833.

6. Pitiot, P., Aldanondo, M., \& Vareilles, E. 2014. Concurrent product configuration and process planning: Some optimization experimental results. Computers in Industry, 65(4), 610-621.

7. Pitiot, P., Aldanondo, M., Vareilles, E., Gaborit, P., Djefel, M., \& Carbonnel, S. 2013. Concurrent product configuration and process planning, towards an approach combining interactivity and optimality. International Journal of Production Research, 51(2), 524-541.

8. Campagna, D., \& Formisano, A. 2013. Product and Production Process Modeling and Configuration. Fundamenta Informaticae, 124(4), 403-425.

9. Lin WANG, Yongjian ZHANG, Shisheng ZHONG. 2016. Typical process discovery based on affinity propagation[J]. Journal of Advanced Mechanical Design, Systems \& Manufacturing, 2016, 10(1), 1-13.

10. Wang Lin, Zhong Shi-sheng, Zhang Yong-jian. 2015. Process configuration based on generative constraint satisfaction problem[J]. Journal of Intelligent Manufacturing, pp. 1-13. 